\title{
Biophysical mechanisms of electroconvulsive therapy-induced volume expansion in the medial temporal lobe: A longitudinal in vivo human imaging study
}

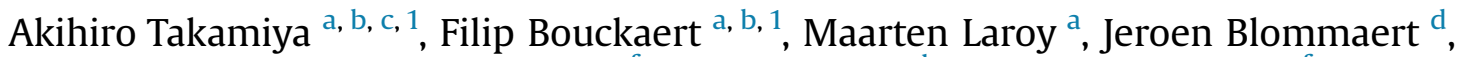 \\ Ahmed Radwan ${ }^{\mathrm{e}}$, Ahmad Khatoun ${ }^{\mathrm{f}}$, Zhi-De Deng ${ }^{\mathrm{g}}$, h, Myles Mc Laughlin ${ }^{\mathrm{f}}$, \\ Wim Van Paesschen ${ }^{i}$, François-Laurent De Winter ${ }^{\text {a, b }}$, Jan Van den Stock ${ }^{\text {a, b }}$,

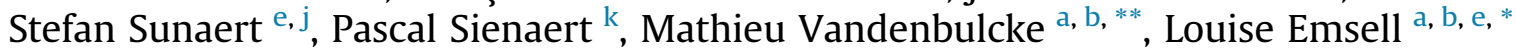 \\ ${ }^{a}$ KU Leuven, Leuven Brain Institute, Department of Neurosciences, Neuropsychiatry, Leuven, Belgium \\ ${ }^{\mathrm{b}}$ Geriatric Psychiatry, University Psychiatric Center KU Leuven, Belgium \\ ${ }^{\mathrm{c}}$ Department of Neuropsychiatry, Keio University School of Medicine, Tokyo, Japan \\ ${ }^{\mathrm{d}}$ KU Leuven, Department of Oncology, Gynaecological Oncology, Leuven, Belgium \\ ${ }^{\mathrm{e}} \mathrm{KU}$ Leuven, Department of Imaging \& Pathology, Translational MRI, Leuven, Belgium \\ ${ }^{\mathrm{f}} \mathrm{KU}$ Leuven, Leuven Brain Institute, Department of Neurosciences, Research Group Experimental Oto-rhino-laryngology, Leuven, Belgium \\ ${ }^{g}$ Noninvasive Neuromodulation Unit, Experimental Therapeutics and Pathophysiology Branch, National Institute of Mental Health, National Institutes of \\ Health, Bethesda, MD, USA \\ ${ }^{\mathrm{h}}$ Department of Psychiatry and Behavioral Sciences, Duke University School of Medicine, Durham, NC, USA \\ ${ }^{\mathrm{i}} \mathrm{KU}$ Leuven, Leuven Brain Institute, Department of Neurosciences, Research Group Experimental Neurology, Leuven, Belgium \\ ${ }^{j}$ Department of Radiology, University Hospitals Leuven (UZ Leuven), Leuven, Belgium \\ ${ }^{\mathrm{k}}$ Academic Center for ECT and Neuromodulation (ACCENT), University Psychiatric Center, KU Leuven, Kortenberg, Belgium
}

\section{A R T I C L E I N F O}

\section{Article history:}

Received 20 April 2021

Received in revised form

23 June 2021

Accepted 23 June 2021

Available online 25 June 2021

\section{Keywords:}

Electroconvulsive therapy

Medial temporal lobe

Morphometry analysis

Electric field

Seizure

\begin{abstract}
A B S T R A C T
Background: Electroconvulsive therapy (ECT) applies electric currents to the brain to induce seizures for therapeutic purposes. ECT increases gray matter (GM) volume, predominantly in the medial temporal lobe (MTL). The contribution of induced seizures to this volume change remains unclear.

Methods: T1-weighted structural MRI was acquired from thirty patients with late-life depression (mean age $72.5 \pm 7.9$ years, 19 female), before and one week after one course of right unilateral ECT. Whole brain voxel-/deformation-/surface-based morphometry analyses were conducted to identify tissue-specific (GM, white matter: WM), and cerebrospinal fluid (CSF) and cerebral morphometry changes following ECT. Whole-brain voxel-wise electric field (EF) strength was estimated to investigate the association of EF distribution and regional brain volume change. The association between percentage volume change in the right MTL and ECT-related parameters (seizure duration, EF, and number of ECT sessions) was investigated using multiple regression.

Results: ECT induced widespread GM volume expansion with corresponding contraction in adjacent CSF compartments, and limited WM change. The regional EF was strongly correlated with the distance from the electrodes, but not with regional volume change. The largest volume expansion was identified in the right MTL, and this was correlated with the total seizure duration.

Conclusions: Right unilateral ECT induces widespread, bilateral regional volume expansion and contraction, with the largest change in the right MTL. This dynamic volume change cannot be explained by the effect of electrical stimulation alone and is related to the cumulative effect of ECT-induced seizures.
\end{abstract}

(C) 2021 The Authors. Published by Elsevier Inc. This is an open access article under the CC BY-NC-ND license (http://creativecommons.org/licenses/by-nc-nd/4.0/).

\footnotetext{
* Corresponding author. KU Leuven, Leuven Brain Institute, Department of Neurosciences, Neuropsychiatry, Leuven, Belgium.

** Corresponding author. Geriatric Psychiatry, University Psychiatric Center KU Leuven, Belgium.

E-mail address: louise.emsell@kuleuven.be (L. Emsell).

1 These authors contributed equally.
} 


\section{Introduction}

Electroconvulsive therapy (ECT) is an established effective treatment for severe depression, especially for older adults [1-4]. Although ECT induces multiple generalized seizures by the application of electric currents through the scalp under general anesthesia, ECT is a safe treatment with a low mortality rate [5], and is not associated with long-term cognitive decline in older patients [6,7] nor with increased risk of developing dementia [8], although some individuals experience cognitive side-effects [6,7].

Gray matter volume (GMV) increase in the medial temporal lobe (MTL), including the hippocampus and amygdala has been consistently reported following ECT [9-11]. Moreover, ECT has been shown to increase dentate gyrus (DG) volume [12-17], a highly neuroplastic region that may be modulated by applied electric currents and seizures [18]. Notably, DG expansion has been associated with clinical improvement $[16,17,19]$, although the clinical relevance of this volume change is unresolved [20-22].

A recent large mega-analysis reported that GMV increase following ECT was not specific to the MTL, but widely distributed across the cortex, with the largest GMV increase in the MTL [23]. Although GMV increase following ECT has been consistently reported, the biophysical mechanism of this phenomenon is still unclear. There are multiple aspects of ECT that could contribute to the observed changes. For example, electrode placement has been associated with the degree of volume change [11,23,24]. The number of ECT sessions may also be an important factor, with more ECTs being associated with greater hippocampal volume increase [11]. Notably, another mega-analysis [25] did not find such association. The strength and distribution of the applied electric field (EF) has also been associated with ECT-related GMV increase in the left MTL, but not in the right MTL [26]. In addition to these ECTrelated parameters, there is another potential candidate which could have an effect on brain structural change following ECT: seizure. Temporal lobe epilepsy is the most frequent form of focal epilepsy [27]. The hippocampus has a lower seizure threshold compared to other brain regions [28] and the amygdala is considered one of the most susceptible brain regions to seizures [29]. Moreover, reversible peri-ictal MRI signal changes are frequently reported [30-32] particularly in the MTL [33] and are hypothesized to reflect the consequences of ictal hemodynamic and metabolic changes that are associated with vasogenic and cytotoxic edema. However, several studies report an absence of increased visible T2 signal or diffusivity changes following ECT, which argues against edema contributing to volumetric change [34-39]. Whilst epileptic seizures arise against a backdrop of neuropathology which limits the utility of epilepsy as a neurobiological model for ECT, there remains parallels between the two conditions which could provide useful insights into post-ictal structural brain changes following ECT. As far as we know, there is only one diffusion tensor imaging (DTI) study reporting that mean seizure duration was associated with decreased fractional anisotropy, a proxy for white matter (WM) organization, following ECT [40].

From a methodological point of view, most previous studies have focused on ECT-related GMV changes using voxel-based morphometry (VBM) [41], surface-based morphometry (SBM) [15,42-44], or region-of-interest (ROI) analysis [11,12,35,36], all of which rely on tissue segmentation. Deformation-based morphometry (DBM) is an alternative way to explore brain structural changes at a macroscopic level [45]. DBM does not rely on tissue segmentation, but utilizes information derived from image registration during spatial normalization. Hence, DBM can be used to investigate global volumetric or shape changes regardless of tissue type [45-47]. In addition, DBM is considered more sensitive to WM change than VBM [48], therefore, combining VBM, SBM and
DBM could provide new insights into the effect of ECT on brain morphometry.

In the present study, we investigate how EF distribution and total seizure duration relate to brain morphometry change following ECT. Based on previous literature [23,26,41], we hypothesized that 1) volume expansion would predominantly occur in the MTL GM, and that 2) MTL volume expansion would be associated with EF and cumulative seizure duration.

\section{Materials and methods}

\section{Participants}

This study was approved by the ethical committee of the Leuven University Hospitals (S5144) and conducted in accordance with the Declaration of Helsinki. Written informed consent was obtained from all participants. Participants were recruited from the inpatient ward at the University Psychiatric Center KU Leuven, Kortenberg, Belgium. Inclusion criteria were a diagnosis of a major depressive disorder according to DSM-IV and age older than 55 years. The diagnoses were confirmed by Mini-International Neuropsychiatric Interview (MINI) assessment. Exclusion criteria were another major psychiatric illness, alcohol or drug dependence, a history of a major neurologic illness (e.g., Parkinson disease, stroke, and dementia) and metal implants precluding MRI. Psychotropic medication was discontinued at least one week before ECT or kept stable from six weeks before ECT and during the ECT course if discontinuation was deemed impossible.

\section{ECT procedure}

ECT was administered twice a week with a constant-current, Thymatron System IV device (Somatics, Venice, FL). Motor and electroencephalographic (EEG) seizures were monitored to ensure adequate duration and quality. Duration of EEG seizure activity was measured by two-channel EEG (Fp1-M1, Fp2-M2, international $10-20$ system). All participants in this study were treated with brief pulse $(0.5 \mathrm{~ms}), 900 \mathrm{~mA}$ current amplitude, right unilateral (RUL) ECT with dosed at six times the initial seizure thresholds, as determined at the first session. ECT was continued until the patients achieved sustained remission defined by MontgomeryÅsberg Depression Rating Scale (MADRS) score $<10$ in two consecutive ratings with a one-week interval or until no more improvement was seen. Patients who did not respond to RUL ECT by the sixth session were switched to bitemporal ECT $(n=5)$. These patients were excluded from this study to ensure sample homogeneity because $\mathrm{EF}$ distribution is related to electrode placement [49]. Etomidate $(0.2 \mathrm{mg} / \mathrm{kg})$ was used for general anesthesia, and succinylcholine ( $1 \mathrm{mg} / \mathrm{kg}$ ) was used to induce muscle relaxation.

\section{MRI data acquisition}

Structural MRI data were acquired within one week before and after the last ECT using a 3T Philips Intera scanner with an eightchannel head coil. 3D magnetization prepared rapid gradient echo (MPRAGE) T1-weighted images were acquired: TR $=9.6 \mathrm{~ms}$, $\mathrm{TE}=4.6 \mathrm{~ms}$, slice thickness $=1.2 \mathrm{~mm}$, voxel size $=0.98 \times 0.98 \times 1.2 \mathrm{~mm}^{3}, 182$ slices. Diffusion MRI data were acquired prior to ECT in 24 participants using a sagittally acquired echo planar imaging sequence with diffusion-weighting, $b=800 \mathrm{~s} /$ $\mathrm{mm}^{2}$, applied along 45 uniformly distributed gradient directions, and included six non-diffusion weighted images, TR/TE $=11000 \mathrm{~ms} /$ $55 \mathrm{~ms}$, 68 slices, voxel size $1.98 \times 1.98 \times 2.2 \mathrm{~mm}^{3}$. 


\section{Morphometry analysis}

The images were visually checked for the presence of any gross motion artifacts or any pathological brain changes preventing further analysis. All pre-processing was conducted using the longitudinal processing pipeline of the Computational Anatomy Toolbox (CAT12) with Statistical Parametric Mapping (SPM)12, including within-subject rigid-body registrations and bias-field corrections. Spatially normalized, modulated tissue-classified images were used to conduct VBM analysis. Each image was smoothed with an $8 \mathrm{~mm}$ isotropic full-width at half-maximum (FWHM) Gaussian kernel. In addition, Jacobian maps from each time point were extracted to conduct DBM analysis. Normalized Jacobian maps were smoothed using two different FWHM Gaussian kernels: an $8 \mathrm{~mm}$ kernel was used in line with the VBM analysis, and a $2 \mathrm{~mm}$ kernel was used to refine the location of ECT-related non-tissue specific volume expansion or contraction. To complement the results from VBM and DBM, we also conducted a surface-based morphometry (SBM) analysis to investigate the effect of ECT on cortical thickness. We used the default pipeline implemented in the CAT12 for SBM. Cortical thickness was estimated using the projection-based thickness method [50]. This algorithm uses tissue segmentation to estimate the WM distance and projects the local maxima to other GM voxels using a neighbor relationship that is described by the WM distance. The vertex-wise thickness measures were resampled and smoothed with a $12 \mathrm{~mm}$ geodesic FWHM Gaussian kernel which is the minimum kernel size recommended in the CAT12 manual.

Paired t-tests were conducted using SPM12 to detect brain morphometry changes following ECT. The statistical threshold was set at a cluster-level family-wise-error (FWE) corrected $\mathrm{p}<0.05$ with an individual voxel-height threshold of $\mathrm{p}<0.001$. To visually compare the results from the three morphometry analyses, the resulting statistical maps were overlaid on the same surface template.

\section{Electric field modeling}

Each individual finite element head model was constructed using the SimNIBS 3.1.2 toolbox (https://simnibs.github.io/simnibs/ build/html/index.html). We used a "headreco" option, which used CAT12/SPM12 for the segmentation of each T1-weighted image and for the creation of the tetrahedral finite element mesh. The head model consisted of five tissue compartments: skin, skull, CSF, GM, and WM, with assigned isotropic conductivities of $0.465 \mathrm{~S} / \mathrm{m}$, $0.010 \mathrm{~S} / \mathrm{m}, 1.654 \mathrm{~S} / \mathrm{m}, 0.276 \mathrm{~S} / \mathrm{m}$, and $0.126 \mathrm{~S} / \mathrm{m}$, respectively [51]. Rectangular electrodes of $4 \times 5 \mathrm{~cm}$ were placed over the C2 and FT8 EEG sites according to the international $10-20$ system to simulate the RUL electrode placement [26]. EF simulation was conducted with an electric current of $1 \mathrm{~mA}$. After normalization to MNI space, the resultant images were multiplied by 900 to be scaled to the current amplitude of the Thymatron device. These processes resulted in subject-specific voxel-wise EF magnitude maps in MNI space. In the model, we assumed that the applied current is static, with the maximum amplitude of the pulse the same as [26].

We also created individual finite element head model incorporating diffusion MRI data to improve the estimation of the EF distribution in 24 participants. The diffusion tensors were estimated using a non-linear least squares approach based on [52] following motion and distortion correction in ExploreDTI [53]. We used dwi2cond command implemented in the SimNIBS, which depends on FMRIB Software Library (FSL) and uses a non-linear registration based on FSL fnirt to transform the tensor imaging to the space of the T1-weighted images. WM anisotropy was incorporated based on conductivity tensors derived from diffusion tensor modeling using a volume normalized mapping approach [54] with maximum eigenvalue of 2 and maximum ratio between eigenvalue of 10 . One dataset was excluded because of registration failure.

\section{Contributing factors to morphometry change following ECT}

To illustrate the voxel-wise distribution of the estimated EF and volume change following ECT, mean images of EF distribution and volume change from all participants were created.

A multiple regression analysis was conducted to identify which ECT-related parameters were associated with the observed brain volume change following ECT. In this regression model, the percentage volume change in the peak cluster based on T-value in the DBM analysis with $2 \mathrm{~mm}$ smoothing kernel was included as a dependent variable. We used the DBM result because ECT could affect both GM and WM, which can be captured by DBM simultaneously. To investigate the effect of seizure properties and electrical stimulation on ECT-related brain volume change, total EEG seizure duration measured by two-channel EEG during the ECT procedure, and the estimated EF in the peak cluster in the DBM analysis were included as independent variables in the regression model. Duration of EEG seizure at the first session was excluded when calculating the total EEG seizure duration because the first session is used to measure each individual's seizure threshold. Missing values of the seizure duration were imputed using the mean value in each participant. The number of ECTs, which has been associated with hippocampal volume increase following ECT [11], was also included in the model. However, the total seizure duration and number of ECTs are highly correlated $(r=0.68, p<0.001)$. Thus, we included residuals of the number of ECTs after regressing out the effect of the total seizure duration to account for the effect of the number of ECTs, which is not explained by the seizure duration. Finally, we conducted a correlation analysis investigating associations between $\mathrm{EF}$ and the effect sizes of regional volume expansion in each ROI defined by the Hammers brain atlas [55] (Supplementary Table 1).

\section{Results}

In the current study, we included 30 older adults with late life depression (age: $72.5 \pm 7.9$, female: $19(63 \%)$ ) who received an average of $11 \pm 3$ right unilateral ECT sessions (range: 4-16). Clinical characteristics of the participants and ECT parameters are presented in Table 1.

Table 1

Clinical characteristics of the participants.

\begin{tabular}{ll}
\hline & ECT patients \\
\hline Number & 30 \\
Age (years) & $72.5(7.9)$ \\
Female & $19(63 \%)$ \\
Psychotic features & $15(50 \%)$ \\
Late-onset depression & $15(50 \%)$ \\
ECT parameters & \\
$\quad$ Number of ECTs & $11(3)$ \\
Total EEG seizure (seconds) & $490(140)$ \\
MADRS at TP1 & $36(8)$ \\
MADRS at TP2 & $8(10)$ \\
Clinical response, $n(\%)$ & $27(90 \%) *$ \\
Clinical remission, $n(\%)$ & $25(83 \%) *$ \\
\hline
\end{tabular}

Continuous variables are expressed as mean (standard deviation).

* Note that we excluded patients who did not respond to RUL ECT and switched to bilateral ECT. 


\section{Morphometry analysis}

VBM showed cortical and subcortical GMV increase, bidirectional WM volume (WMV) change, and CSF decrease following ECT. The WMV decrease occurred mainly adjacent to the regions of GMV increase, and the WMV increase occurred mainly in the periventricular regions (Fig. 1).

When using an 8-mm smoothing kernel, DBM showed widespread volume expansion in both GM and WM with corresponding contraction in adjacent CSF compartments. DBM with 2-mm smoothing kernel revealed that the volume expansion was mainly located in the GM, and that limited WM volume change was observed
(Fig. 2). The largest volume expansion was identified in the right MTL surviving voxel-level FWE-correction $\mathrm{p}<0.05$ (Supplementary Fig. 1).

SBM analysis showed widespread cortical thickness increase bilaterally (Fig. 3A). To visually compare the results of the morphometry analyses, all morphometry results were overlaid on the same surface template (Fig. 3).

Voxel-wise electric field estimation and brain shape change following ECT

Mean voxel-wise EF distribution and volume expansion following ECT are presented in Fig. 4, and the EF distribution is also
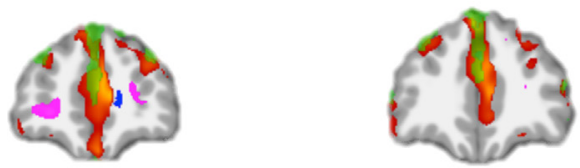

$$
y=50
$$
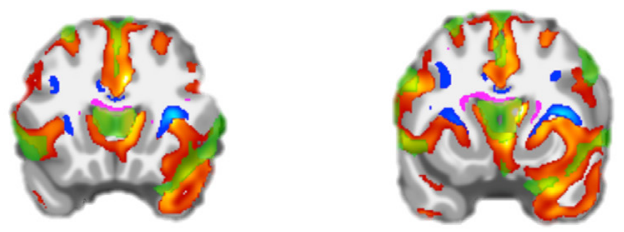

$$
y=18
$$
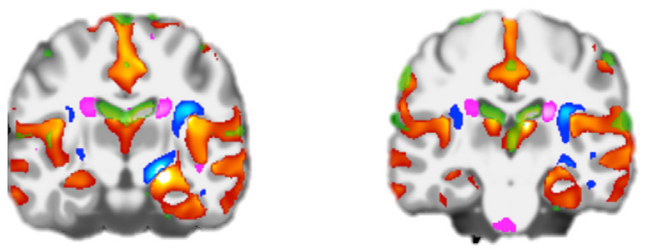

$$
y=-14
$$
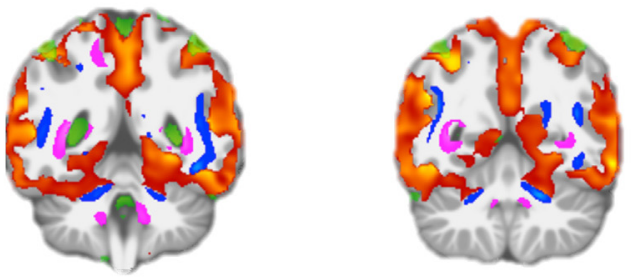

$$
y=-46
$$

$\mathrm{L}$

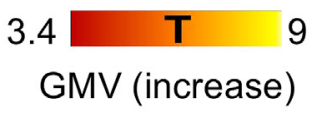

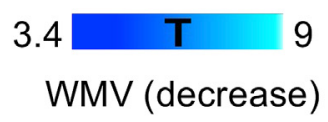
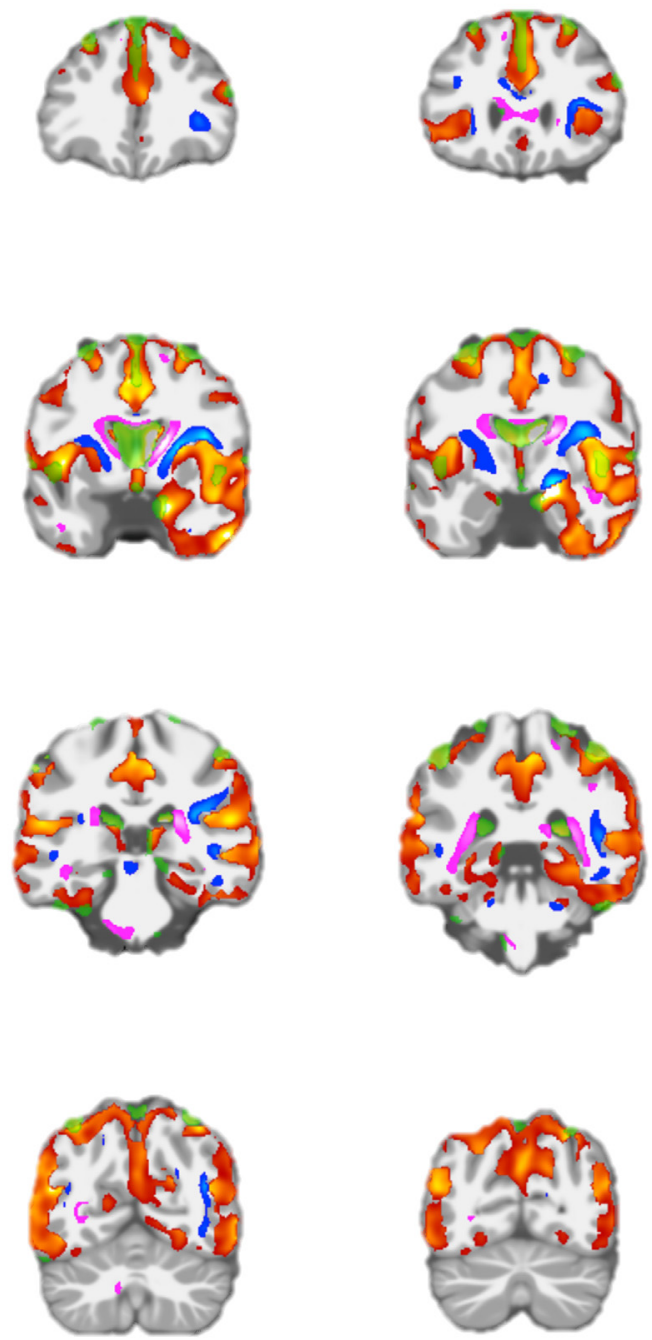

$\mathrm{R}$

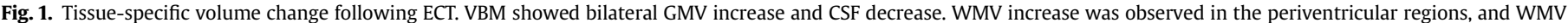

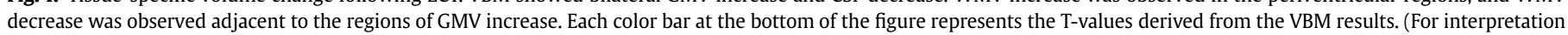
of the references to color in this figure legend, the reader is referred to the Web version of this article.) 

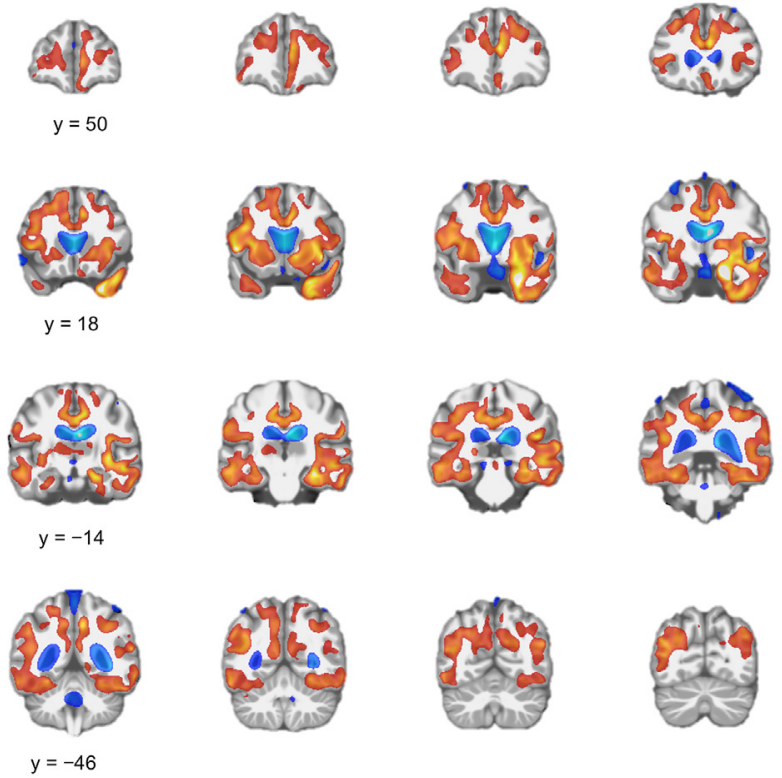

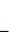

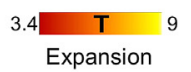

$\mathrm{R}$

B

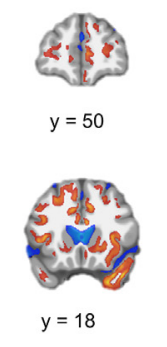

DBM (2 mm smoothing)
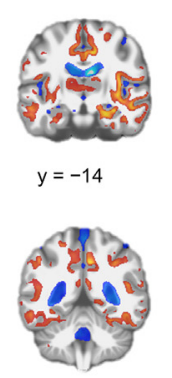

$y=-46$

L

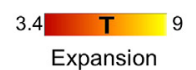

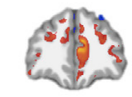
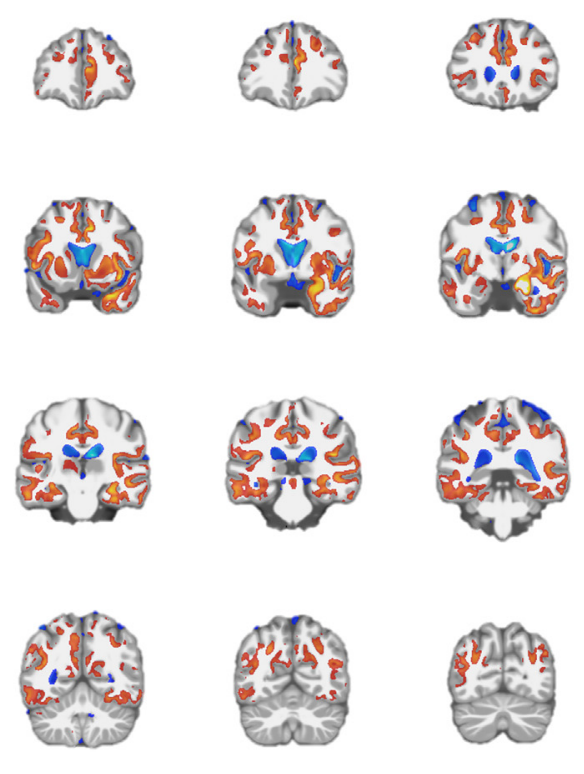

$\mathrm{R}$

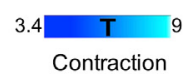

was a correlation between the EF and effect size of regional volume expansion (Spearman's rho $=0.27, \mathrm{p}=0.04$ ), but no correlations when analyzing each hemisphere separately (Supplementary Fig. 2). The EF strongly correlated with the distance from the brain regions near the electrodes (i.e., right precentral gyrus and right superior temporal gyrus) (Supplementary Fig. 3).

\section{Association between ECT-related parameters and volume expansion}

There was a trend toward positive correlation between the total seizure duration and the MTL volume increase $(r=0.34, \mathrm{df}=28$, $\mathrm{p}=0.066$ ), and there was no correlation between the EF and the MTL volume change $(r=0.13, d f=28, p=0.50)$. In the multiple regression model of the full sample, the total EEG seizure duration was associated with the percentage volume expansion in the right MTL ( $p=0.039$, Table 2). Even after controlling for age or medication usage, the results did not change (Supplementary Tables 3 and 4$)$. In the subgroup $(\mathrm{n}=23$ ) incorporating DTI derived anisotropy data to improve the EF model, the result of the regression analyses was similar: the total EEG seizure duration was associated with the percentage volume expansion in the right MTL ( $\mathrm{p}=0.045$, Supplementary Table 5).

Because the GEMRIC mega-analysis reported significant correlations between EF and ECT-related brain volume increases in the left hippocampus and amygdala [26], we also investigated the relationships among EF, seizures, and volume changes in the left hippocampus/amygdala as a supplementary analysis. In simple correlation analyses, we found a significant correlation between the EF and left hippocampal volume expansion $(\mathrm{r}=0.37, \mathrm{df}=28$, $\mathrm{p}=0.04$ ), and a correlation between the EF and left amygdala volume expansion $(\mathrm{r}=0.35, \mathrm{df}=28, \mathrm{p}=0.056)$. In multiple regression models including total EEG seizure duration and the residuals of the number of ECTs, these associations remained (Supplementary Table 6).

\section{Discussion}

Our results provide evidence of dynamic volume changes following ECT, suggesting that GM volume expansion asserted a mass effect on adjacent tissue with compensatory volume decrease in CSF-filled spaces. The effect on WM was less pronounced, and the volumetric changes observed with VBM could be interpreted as a displacement based on the DBM results. Moreover, the largest volume expansion in the right MTL did not correlate with the EF distribution, but with the total seizure duration over the ECT course.

\section{Differential effect of ECT on gray and white matter}

In this study, DBM revealed that brain deformation changes following ECT were observed mainly in the GM and CSF compartments, but less change was identified in the WM. This discrepancy is interesting as it argues against a uniform effect of ECT on brain tissue. Although the effect of ECT on WM has been reported in several DTI studies [40,56-58], these results were inconsistent, and some were contradictory. Some may argue that T1-weighted imaging lacks sensitivity for detecting WM changes. However, previous DBM analyses have reported epilepsy-related [59] and chemotherapy-related [60] WM changes. Therefore, the effect of ECT on WM might be too small to detect using DBM analysis or ECT might not cause macroscopic WM change (e.g., axonal swelling). Although our VBM analysis revealed a bidirectional WMV change pattern, these changes were observed mainly next to expanded GM or contracted CSF-filled spaces (Fig. 1). In addition, there were significant correlations among the degree of GMV increase, WMV overlaid on the surface template (Fig. 3D). Top-ranked brain regions exposed to the highest EF and brain regions showing the largest volume expansion are presented in Supplementary Table 2. There 
A

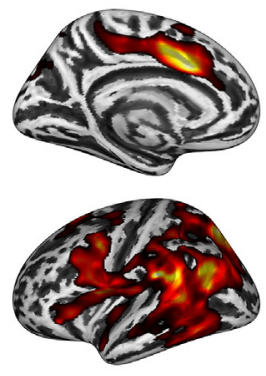

C

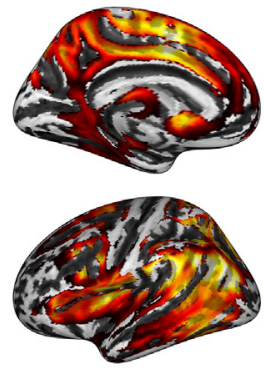

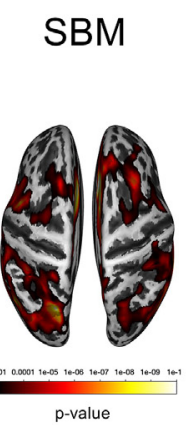

VBM

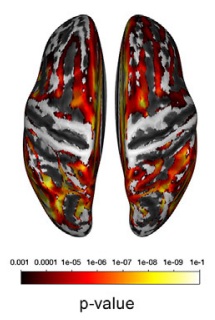

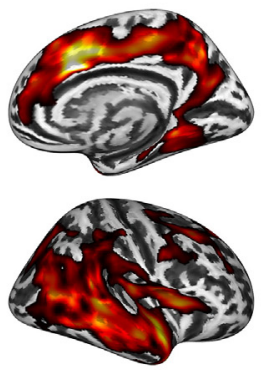

B

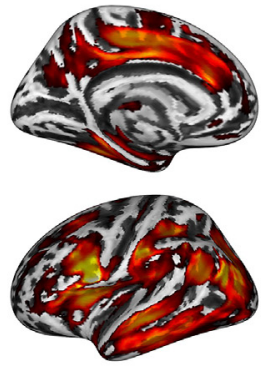

D
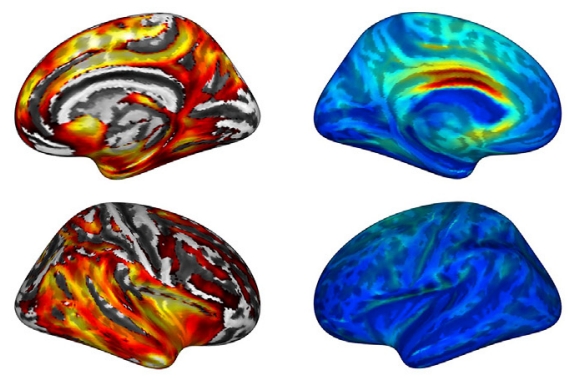
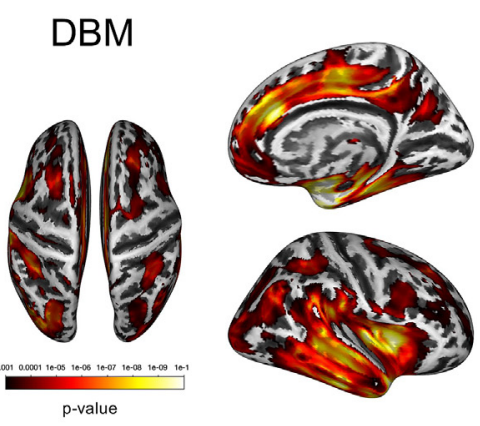

Electric Field

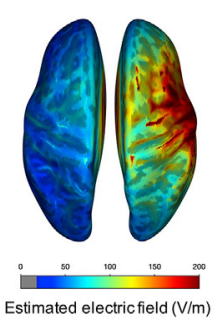

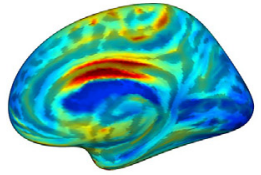
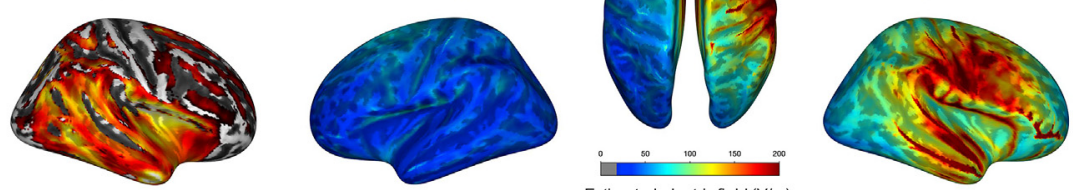

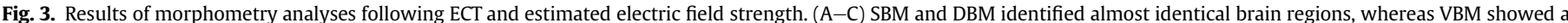

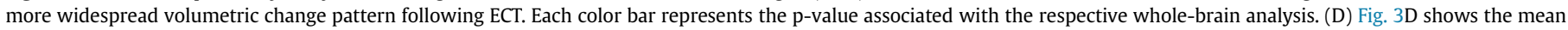

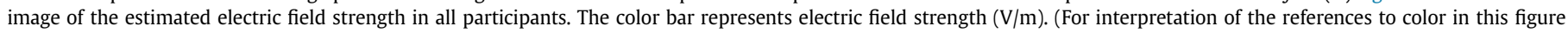
legend, the reader is referred to the Web version of this article.)

increase/decrease, and CSF decrease (Supplementary Fig. 4). Taken together, these findings suggest that the WMV changes detected by VBM may reflect WM displacement due to adjacent expanded GM.

In line with the literature [11,12,15,23,35,41-44,61], all morphometry analyses consistently found both GM volume and cortical thickness increase following ECT, with the largest change in the right MTL. Multiple mechanisms may give rise to this GM expansion, including neuroplastic changes, changes in intracellular and extracellular fluid compartments (e.g., due to edema), immunological activation and altered cerebral blood flow [18,62]. In general, more than $80 \%$ of the cortical volume is made up of neuropil (i.e., the axons, dendrites, synapses, glial cell processes and microvasculature), and the rest is made up of neuronal cell bodies, blood vessels and glial cell bodies [63]. The number of neurons in the cortex is considered to remain largely constant [64], therefore, cortical volumetric expansion and cortical thickness increase may reflect an increase in dendrites and synapses [63]. Neurogenesis has been postulated as a potential mechanism underlying volume increase in the hippocampus [18], but the magnitude of the MTL volume expansion $(>5 \%)$ and cortical extent of GM change suggests other, additional neuroplastic mechanisms also contribute to the volume increase. Synaptic plasticity might be accompanied by growth of both capillaries and glia [66], perhaps to support the increased energy demand of new synapses [67]. Indeed, electroconvulsive seizures (ECS), an animal model of ECT, has been shown to alter the number, morphology, and activation of glial cells [68], including astrocytes [69]. Interestingly, astrocytic activation may change the MRI T1-relaxation time manifesting as GMV increase [70], and vascular changes could also affect GMV estimation because of the relatively similar relaxation time constants between arterial blood and GM [71].

Another consequence of vascular and immunological changes is edema. Volume increase in the peri-ictal phase following epileptic seizure has been detected by other MRI sequences (e.g., T2- weighted images) and attributed to cellular edema [72]. However, DTI studies have consistently reported decreased water diffusivity in the MTL following ECT [36-38]. Whilst reduced mean diffusivity could theoretically reflect acute cytotoxic edema, a recent multicontrast quantitative MRI study reported that GMV increase following ECT was not paralleled by an increase in proton density, a proxy for tissue water content. These findings suggest that neither vasogenic nor cytotoxic edema are likely to be the primary contributor to MTL volume increase following ECT [39].

\section{Effect of electrical stimulation on regional volume change following $E C T$}

We found that regional EF strength, which is a proxy for the effect of direct electrical stimulation rather than the seizure activity, was the highest in the brain regions near the stimulus electrodes (i.e., GM of the right pre/post central gyrus and the right superior temporal gyrus with the greatest magnitude in the adjacent WM), in line with a previous study [49]. The regional EF was strongly correlated with the Euclidean distance from these brain regions (Supplementary Fig. 3), but not with the regional volume change when analyzing the ROIs in each hemisphere separately. The right pre/post central gyrus did not show statistically significant volume or thickness change even with the exposure to the highest EF. Indeed, the spatial distribution of the volume changes are more bilaterally symmetric compared to the lateralized EF distribution induced by RUL ECT (Fig. 3), although the volume expansion near the hippocampus appear more lateralized to the right hemisphere (Fig. 4). Interestingly, MTL volumetric increase has been reported following transcranial magnetic stimulation in several studies [72,73], but not all [74], and it has also been reported following vagus nerve stimulation [75]. This evidence suggests that state-dependent clinical change regardless of treatment modalities can be associated with the MTL volume increase. In addition, these 
A
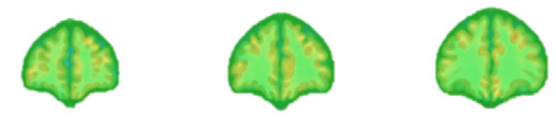

$y=40$
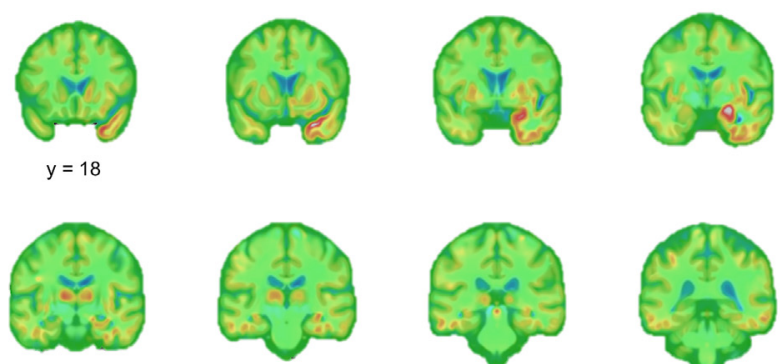

$y=-14$
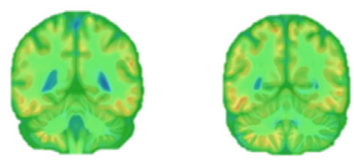

$y=-46$

$\mathrm{L}$

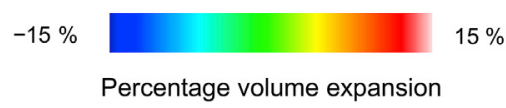

B
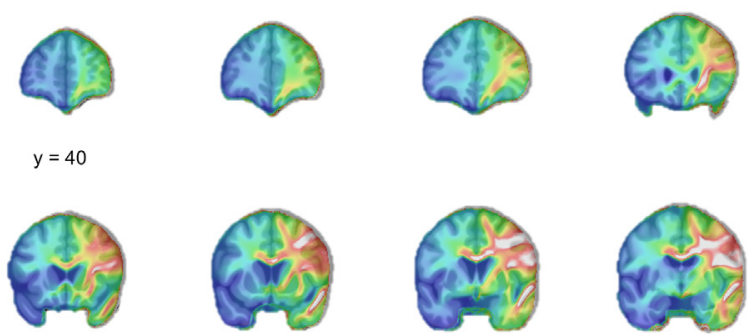

$y=18$
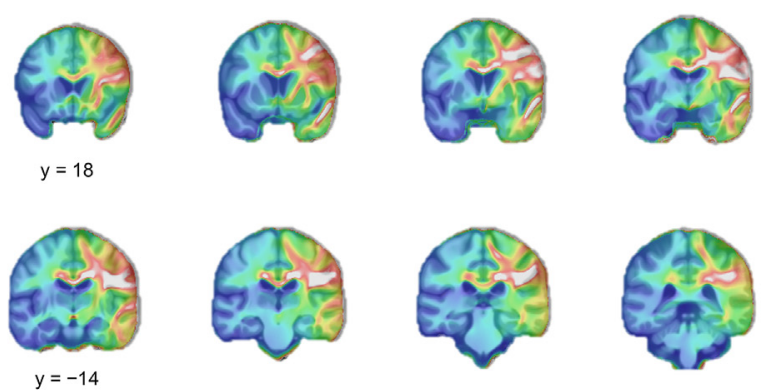

$\mathrm{R}$

$\%$
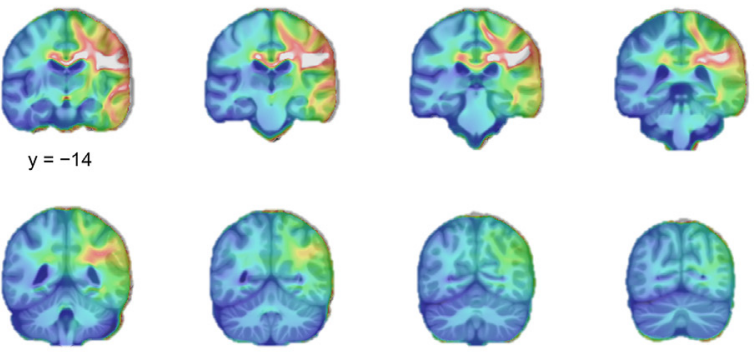

$y=-46$

$\mathrm{L}$
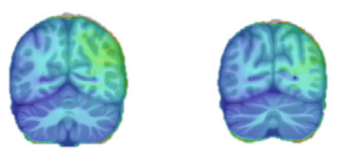

$\mathrm{R}$

0

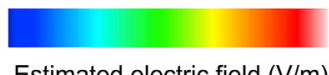

200

Fig. 4. Different patterns of the volume change following ECT and estimated electric field strength. (A) Fig. 4A shows the mean image of the DBM results. The largest volume expansion was observed in the right medial temporal lobe. The color bar represents percentage volume expansion. (B) Fig. 4B shows the mean image of the estimated electric field. The color bar represents electric field strength $(\mathrm{V} / \mathrm{m})$ ). (For interpretation of the references to color in this figure legend, the reader is referred to the Web version of this article.)

results suggest that the topography of the applied electrical field alone cannot explain the regional volume change following ECT, especially the largest volume expansion in the right MTL.
Table 2

ECT parameters associated with percentage volume expansion in the right MTL.

\begin{tabular}{lllll}
\hline & B & SE & $\beta$ & p-value \\
\hline Total EEG seizure duration & 0.01 & 0.006 & 0.39 & 0.039 \\
Electric field & 0.11 & 0.09 & 0.24 & 0.20 \\
Number of ECTs (residuals)* & 0.38 & 0.38 & 0.18 & 0.33 \\
\hline
\end{tabular}

B: non-standardized coefficient; SE: standard error; $\beta$ : standardized coefficient.

* The number of ECTs (residuals) is the information about the number of ECTs that is not explained by the total EEG seizure duration, hence due to the high correlation $(r=0.68, p<0.001)$ all information about the number of ECTs is included in this regression model.

Our results and arguments do not fully contradict a recent mega-analysis (which also included data from the present study) [26]. We replicated the associations between EF, left hippocampal and left amygdala volume expansion despite several methodological differences between our analyses.

\section{Effect of seizure on regional volume change following ECT}

In the present study, the right MTL volume expansion was associated with the total EEG seizure duration, i.e., the sum of the seizure duration per session. ECT-induced seizures are focal to bilateral tonic-clonic seizures, involving certain specific cortical and subcortical brain regions, while other brain regions are less affected [76]. Moreover, RUL ECT-induced seizures are not always symmetrical, but can be asymmetrical with higher EEG amplitude on the right-side during seizures [77]. In this context, the origin and propagation pattern of ECT-induced seizure is relevant. The MTL or precentral gyrus may be the origin of ECT-induced seizure because of their lower seizure threshold relative to other brain regions [28]. We found large volume expansion in the right amygdala, which is in line with a previous mega-analysis [23]. Interestingly, the amygdala is often chosen as a target for kindling in preclinical research due to its susceptibility for seizure generation [78]. One animal study including 25 cats reported that bilateral ECT-induced seizures initiated in the sensorimotor cortex resulted in the largest discharge in the hippocampus. Moreover, the hippocampal seizure activity continued longer than in other brain regions and propagated to functionally connected regions, such as the amygdala [79]. Another study which investigated epileptic seizure propagation from the hippocampus reported intense activation of the hippocampus resulting from re-entrant seizure activity, and hippocampal activity throughout the course of the seizure in contrast to the cortical structures [80]. Our results suggest that the estimated EF was large enough to elicit neuronal depolarization in almost all brain regions (Supplementary Table 2; Supplementary Figs. 2 and 3 ), therefore other brain regions or several brain regions could be the origin(s) of ECT-induced seizure.

The relationship between seizures and brain volumetric change is complicated. There are several differences between ECT-induced seizures and disease-related epileptic seizures. For example, the effect of epileptic seizures on brain structure may vary across brain regions and includes volume reduction in the MTL and thalamus [81], and volume increase in the amygdala [82], which may normalize following successful antiepileptic treatment [83]. According to preclinical studies, epileptic seizures result in the generation of new neurons characterized by structural and functional abnormalities [84], which is in contrast with ECS-treated animals, where no new-born neurons in the hilus (ectopic) were observed $[85,86]$. ECS induces sprouting of the granule cell mossy fiber pathway in the hippocampus without generation of abnormal connectivity and does not lead to the appearance of spontaneous epileptic activity. ECS-induced sprouting occurs in the absence of 
neuronal loss, indicating that sprouting is not a compensatory response to cell death [87].

\section{Limitations}

Measuring the applied current and seizure duration, and investigating their relationships is not trivial. First, we measured seizure duration using two-channel EEG in a clinical setting. Second, we used the summed seizure duration to assess the cumulative effect of seizures across the whole ECT course. This is because the seizure duration decreased later in the course of ECT, which may be due to increased seizure threshold (Supplementary Fig. 5). This heterogeneity was not accounted for in our models. In addition, whilst we attempted to statistically disentangle the effects of total seizure duration from the number of ECT sessions, the total seizure duration itself inherently includes information about the number of ECTs. Our model does not deny the importance of the number of ECTs in the context of the right MTL volume expansion. However, this relationship is complicated as the number of ECTs is correlated with opposing seizure-related parameters (Supplementary Fig. 5). As our research question focused on the effect of seizures, we prioritized this in our analysis and reporting, using the residuals to regress out the effect of seizure on the number of treatments. However, the same analysis including the total EEG seizure duration after regressing out the effect of the number of ECTs, would show treatment number to be correlated with right MTL volume expansion. There are also likely other factors beyond seizure duration that contribute to these relationships, for example, ictal seizure morphology, including temporal coherence, power, post-ictal suppression, which were not accounted for in our model. As in the mega-analysis [26], the EF model accounted for spatial aspect (including current amplitude) but not temporal information of the pulse train (e.g., pulse width and polarity, frequency, and number of pulses). Parameters of the temporal pulse sequence such as stimulus train frequency and duration can significantly affect seizure duration and postictal suppression [88]. These parameters are typically reported as part of an aggregate measure of dosing, i.e., stimulus charge, which have been shown to impact seizure expression [89]. Future studies should consider the effects of individual stimulus parameters on seizure expression and brain volume change. In addition, our statistical model considers EF and seizure to be independent, however this distinction is artificial given that the applied EF may cause seizure. Given the limited data available to us, we were unable to use more complex models to account for this relationship. There is therefore a need to investigate the role of the EF on seizure initiation and subsequent propagation. Given these limitations, our findings relating seizure duration and volume change should be considered preliminary. We anticipate however that these initial insights will form the basis for future work which should consider the effects of individual stimulus parameters on seizure expression as well as seizure characteristics on brain volume change following ECT.

\section{Conclusion}

ECT induced widespread GM expansion with volume contraction in the CSF compartments. The largest volume expansion in the right MTL could not be explained by the effect of electrical stimulation alone and was associated with total seizure duration during a course of therapy.

\section{Declaration of interest}

None.

\section{CRediT authorship contribution statement}

Akihiro Takamiya: Conceptualization, Methodology, Formal analysis, Investigation, Writing - original draft, Visualization. Filip Bouckaert: Conceptualization, Investigation, Writing - review \& editing, Supervision, Funding acquisition. Maarten Laroy: Methodology, Writing - review \& editing. Jeroen Blommaert: Methodology, Writing - review \& editing. Ahmed Radwan: Methodology, Writing - review \& editing. Ahmad Khatoun: Methodology, Writing - review \& editing. Zhi-De Deng: Methodology, Writing - review \& editing. Myles Mc Laughlin: Methodology, Writing - review \& editing. Wim Van Paesschen: Writing review \& editing. François-Laurent De Winter: Investigation, Writing - review \& editing. Jan Van den Stock: Writing - review \& editing. Stefan Sunaert: Writing - review \& editing. Pascal Sienaert: Writing - review \& editing. Mathieu Vandenbulcke: Conceptualization, Writing - review \& editing, Supervision, Project administration, Funding acquisition. Louise Emsell: Conceptualization, Methodology, Investigation, Writing - review \& editing, Supervision, Project administration, Funding acquisition.

\section{Acknowledgements}

This study was supported by the Research Foundation Flanders (FWO) project G074609 (M.Vandenbulcke), G0C0319 N (M. Vandenbulcke, F. Bouckaert, L. Emsell), Research Grant Old Age Psychiatry UZ Leuven R94859 (M. Vandenbulcke), KU Leuven C24/18/ 095 (M. Vandenbulcke, F. Bouckaert, J. Van den Stock, L. Emsell) and KU Leuven Sequoia Fund. A. Takamiya has received grants from Keio University Medical Science Fund and Kanae Foundation for the Promotion of Medical Science to study in Belgium and is supported by AMED (Grant Number: JP20dm0307102h0003). M. Laroy and J. Blommaert are aspirant researchers for the Research Foundation Flanders (FWO, grant no. 11B9919 N, 1168821 N). Z.-D. Deng is supported by the Intramural Research Program of the National Institute of Mental Health, National Institutes of Health (ZIAMH002955).

\section{Appendix A. Supplementary data}

Supplementary data to this article can be found online at https://doi.org/10.1016/j.brs.2021.06.011.

\section{References}

[1] Kellner CH, Greenberg RM, Murrough JW, Bryson EO, Briggs MC, Pasculli RM. ECT in treatment-resistant depression. Am J Psychiatr 2012:169:1238-44.

[2] Kellner CH, Husain MM, Knapp RG, McCall WV, Petrides G, Rudorfer MV, et al. Rightunilateral ultrabrief pulse ect in geriatric depression: phase 1 of the pride study. Am J Psychiatr 2016;173:1101-9. https://doi.org/10.1176/ appi.ajp. 2016.15081101.

[3] Dols A, Bouckaert F, Sienaert P, Rhebergen D, Vansteelandt K, ten Kate M, et al. Early- and late-onset depression in late life: a prospective study on clinical and structural brain characteristics and response to electroconvulsive therapy. Am J Geriatr Psychiatr 2017;25:178-89. https://doi.org/10.1016/ j.jagp.2016.09.005.

[4] Van Diermen L, Van Den Ameele S, Kamperman AM, Sabbe BCG, Vermeulen T, Schrijvers $D$, et al. Prediction of electroconvulsive therapy response and remission in major depression: meta-analysis. Br J Psychiatry 2018;212: 71-80. https://doi.org/10.1192/bjp.2017.28.

[5] Tørring N, Sanghani SN, Petrides G, Kellner CH, Østergaard SD. The mortality rate of electroconvulsive therapy: a systematic review and pooled analysis. Acta Psychiatr Scand 2017;135:388-97. https://doi.org/10.1111/acps.12721.

[6] Obbels J, Verwijk E, Vansteelandt K, Dols A, Bouckaert F, Schouws S, et al. Long-term neurocognitive functioning after electroconvulsive therapy in patients with late-life depression. Acta Psychiatr Scand 2018;138:223-31. https://doi.org/10.1111/acps.12942.

[7] Obbels J, Vansteelandt K, Bouckaert F, Dols A, Stek M, Verwijk E, et al. Neurocognitive functioning after electroconvulsive therapy in late-life depression: a 4-year prospective study. Acta Psychiatr Scand 2021;143:141-50. https:// doi.org/10.1111/acps.13252. 
[8] Osler M, Rozing MP, Christensen GT, Andersen PK, Jørgensen MB. Electroconvulsive therapy and risk of dementia in patients with affective disorders: a cohort study. The Lancet Psychiatry 2018;5:348-56. https://doi.org/10.1016/ S2215-0366(18)30056-7.

[9] Takamiya A, Chung JK, Liang KC, Graff-Guerrero A, Mimura M, Kishimoto T. Effect of electroconvulsive therapy on hippocampal and amygdala volumes: systematic review and meta-analysis. $\mathrm{Br} \mathrm{J}$ Psychiatry 2018;212:19-26. https://doi.org/10.1192/bjp.2017.11.

[10] Gbyl K, Videbech P. Electroconvulsive therapy increases brain volume in major depression: a systematic review and meta-analysis. Acta Psychiatr Scand 2018;138:180-95. https://doi.org/10.1111/acps.12884.

[11] Oltedal L, Narr KL, Abbott C, Anand A, Argyelan M, Bartsch H, et al. Volume of the human Hippocampus and clinical response following electroconvulsive therapy. Biol Psychiatr 2018;84:574-81. https://doi.org/10.1016/ j.biopsych.2018.05.017.

[12] Abbott CC, Jones T, Lemke NT, Gallegos P, McClintock SM, Mayer AR, et al. Hippocampal structural and functional changes associated with electroconvulsive therapy response. Transl Psychiatry 2014;4:e483-7. https://doi.org/ 10.1038/tp.2014.124.

[13] Cao B, Luo Q, Fu Y, Du L, Qiu T, Yang X, et al. Predicting individual responses to the electroconvulsive therapy with hippocampal subfield volumes in major depression disorder. Sci Rep 2018;8:1-8. https://doi.org/10.1038/s41598018-23685-9.

[14] Takamiya A, Plitman E, Chung JK, Chakravarty M, Graff-Guerrero A, Mimura M, et al. Acute and long-term effects of electroconvulsive therapy on human dentate gyrus. Neuropsychopharmacology 2019:1805-11. https:// doi.org/10.1038/s41386-019-0312-0.

[15] Gryglewski G, Baldinger-Melich P, Seiger R, Godbersen GM, Michenthaler P, Klöbl M, et al. Structural changes in amygdala nuclei, hippocampal subfields and cortical thickness following electroconvulsive therapy in treatmentresistant depression: longitudinal analysis. $\mathrm{Br} \mathrm{J}$ Psychiatry 2019;214: 159-67. https://doi.org/10.1192/bjp.2018.224.

[16] Nuninga JO, Mandl RCW, Boks MP, Bakker S, Somers M, Heringa SM, et al. Volume increase in the dentate gyrus after electroconvulsive therapy in depressed patients as measured with 7T. Mol Psychiatr 2020;25:1559-68. https://doi.org/10.1038/s41380-019-0392-6.

[17] Gbyl K, Rostrup E, Raghava JM, Andersen C, Rosenberg R, Larsson HBW, et al. Volume of hippocampal subregions and clinical improvement following electroconvulsive therapy in patients with depression. Prog NeuroPsychopharmacol Biol Psychiatry 2021;104:110048. https://doi.org/10.1016/ j.pnpbp.2020.110048.

[18] Bouckaert F, Sienaert P, Obbels J, Dols A, Vandenbulcke M, Stek M, et al. ECT: its brain enabling effects: a review of electroconvulsive therapy-induced structural brain plasticity. J ECT 2014;30:143-51. https://doi.org/10.1097/ YCT.0000000000000129.

[19] Takmaiya A, Nuninga JO, Mandl RCW, Sommer IEC, Mimura M, Kishimoto T. Volume increase of the dentate gyrus induced by electroconvulsive therapy: shedding light on the clinical relevance of plasticity in the Hippocampus. J ECT 2019;35:e57-8. https://doi.org/10.1097/YCT.0000000000000630.

[20] Nuninga JO, Mandl RCW, Sommer IEC. Clinical relevance of brain changes after electroconvulsive therapy: is there really No link at all? Biol Psychiatr 2020. https://doi.org/10.1016/j.biopsych.2020.04.030. S0006-3223:31639-5.

[21] Ousdal OT, Gjestad R, Oltedal L. Reply to: clinical relevance of brain changes after electroconvulsive therapy: is there really No link at all? Biol Psychiatr 2020. https://doi.org/10.1016/j.biopsych.2020.05.031. S0006-3223:31638-3.

[22] Laroy M, Vansteelandt K, Emsell L, Van Den Stock J, Vandenbulcke M, Bouckaert F. Response to volume increase in the dentate gyrus induced by electroconvulsive therapy shedding light on the clinical relevance of plasticity in the Hippocampus. 2020. https://doi.org/10.1097/YCT.0000000000000715.

[23] Ousdal OT, Argyelan M, Narr KL, Abbott C, Wade B, Vandenbulcke M, et al. Brain changes induced by electroconvulsive therapy are broadly distributed. Biol Psychiatr 2020;87:451-61. https://doi.org/10.1016/ j.biopsych.2019.07.010.

[24] Cano M, Lee E, Cardoner N, Martínez-Zalacaín I, Pujol J, Makris N, et al. Brain volumetric correlates of right unilateral versus bitemporal electroconvulsive therapy for treatment-resistant depression. J Neuropsychiatry Clin Neurosci 2019;31:152-8. https://doi.org/10.1176/appi.neuropsych.18080177.

[25] Sartorius A, Demirakca T, Böhringer A, Clemm von Hohenberg C, Aksay SS, Bumb JM, et al. Electroconvulsive therapy induced gray matter increase is not necessarily correlated with clinical data in depressed patients. Brain Stimul 2019;12:335-43. https://doi.org/10.1016/j.brs.2018.11.017.

[26] Argyelan M, Oltedal L, Deng Z De, Wade B, Bikson M, Joanlanne A, et al. Electric field causes volumetric changes in the human brain. Elife 2019;8: 1-20. https://doi.org/10.7554/eLife.49115.

[27] Blair RDG. Temporal lobe epilepsy semiology. Epilepsy Res Treat 2012:1-10. https://doi.org/10.1155/2012/751510. 2012

[28] Penfield W, Jasper H. Epilepsy and the functional anatomy of the human brain. Boston, Mass: Little, Brown \& Co Inc; 1954.

[29] Cota VR, Drabowski BMB, Oliveira JC de, Moraes MFD. The epileptic amygdala: toward the development of a neural prosthesis by temporally coded electrical stimulation. J Neurosci Res 2016;94:463-85.

[30] Cole AJ. Status epilepticus and periictal imaging. Epilepsia 2004;45:72-7. https://doi.org/10.1111/j.0013-9580.2004.04014.x.

[31] Cianfoni A, Caulo M, Cerase A, Della Marca G, Falcone C, Di Lella GM, et al. Seizure-induced brain lesions: a wide spectrum of variably reversible MRI abnormalities. Eur J Radiol 2013;82:1964-72. https://doi.org/10.1016/ j.ejrad.2013.05.020.

[32] Williams JA, Bede P, Doherty CP. An exploration of the spectrum of peri-ictal MRI change; a comprehensive literature review. Seizure 2017;50:19-32. https://doi.org/10.1016/j.seizure.2017.05.005.

[33] Chatzikonstantinou A, Gass A, Förster A, Hennerici MG, Szabo K. Features of acute DWI abnormalities related to status epilepticus. Epilepsy Res 2011;97: 45-51. https://doi.org/10.1016/j.eplepsyres.2011.07.002.

[34] Kunigiri G, Jayakumar PN, Janakiramaiah N, Gangadhar BN. MRI T(2) relaxometry of brain regions and cognitive dysfunction following electroconvulsive therapy. Indian J Psychiatr 2007;49:195-9. https://doi.org/10.4103/00195545.37321.

[35] Nordanskog P, Dahlstrand U, Larsson MR, Larsson EM, Knutsson L, Johanson A Increase in hippocampal volume after electroconvulsive therapy in patients with depression: a volumetric magnetic resonance imaging study. J ECT 2010;26:62-7. https://doi.org/10.1097/YCT.0b013e3181a95da8.

[36] Jorgensen a, Magnusson P, Hanson LG, Kirkegaard T, Benveniste H, Lee H, et al. Regional brain volumes, diffusivity, and metabolite changes after electroconvulsive therapy for severe depression. Acta Psychiatr Scand 2016: 133154-64. https://doi.org/10.1111/acps.12462.

[37] Yrondi A, Nemmi F, Billoux S, Giron A, Sporer M, Taib S, et al. Significant decrease in hippocampus and amygdala mean diffusivity in treatmentresistant depression patients who respond to electroconvulsive therapy. Front Psychiatr 2019;10:1-8. https://doi.org/10.3389/fpsyt.2019.00694.

[38] Nuninga JO, Mandl RCW, Froeling M, Siero JCW, Somers M, Boks MP, et al. Vasogenic edema versus neuroplasticity as neural correlates of hippocampal volume increase following electroconvulsive therapy. Brain Stimul 2020;13: 1080-6. https://doi.org/10.1016/j.brs.2020.04.017.

[39] Gyger L, Ramponi C, Mall JF, Swierkocz-Lenart k, Stoyanov D, Lutti A, et al. Temporal trajectory of brain tissue property changes induced by electroconvulsive therapy. Neuroimage 2021. https://doi.org/10.1016/j.neuroimage.2021.117895 (in press).

[40] Repple J, Meinert S, Bollettini I, Grotegerd D, Redlich R, Zaremba D, et al. Influence of electroconvulsive therapy on white matter structure in a diffusion tensor imaging study. Psychol Med 2020;50:849-56. https://doi.org/10.1017/ S0033291719000758.

[41] Bouckaert F, De Winter FL, Emsell L, Dols A, Rhebergen D, Wampers M, et al. Grey matter volume increase following electroconvulsive therapy in patients with late life depression: a longitudinal MRI study. J Psychiatry Neurosci 2016;41:105-14. https://doi.org/10.1503/jpn.140322.

[42] Pirnia T, Joshi SH, Leaver AM, Vasavada M, Njau S, Woods RP, et al. Electroconvulsive therapy and structural neuroplasticity in neocortical, limbic and paralimbic cortex. Transl Psychiatry 2016;6:e832-8. https://doi.org/10.1038/ tp.2016.102.

[43] van Eijndhoven P, Mulders P, Kwekkeboom L, van Oostrom I, van Beek M, Janzing J, et al. Bilateral ECT induces bilateral increases in regional cortical thickness. Transl Psychiatry 2016;6:e874. https://doi.org/10.1038/ tp.2016.139.

[44] Yrondi A, Nemmi F, Billoux S, Giron A, Sporer M, Taib S, et al. Grey Matter changes in treatment-resistant depression during electroconvulsive therapy. J Affect Disord 2019;258:42-9. https://doi.org/10.1016/j.jad.2019.07.075.B.

[45] Ashburner J, Hutton C, Frackowiak R, Johnsrude I, Price C, Friston K. Identifying global anatomical differences: deformation-based morphometry. Hum Brain Mapp 1998;6:348-57. https://doi.org/10.1002/(SICI)1097-0193(1998) 6:5/6<348::AID-HBM 4> 3.0.CO;2-P.

[46] Despotović I, Goossens B, Philips W. MRI segmentation of the human brain: challenges, methods, and applications. Comput Math Methods Med 2015. https://doi.org/10.1155/2015/450341. 2015.

[47] Gaser C, Nenadic I, Buchsbaum BR, Hazlett EA, Buchsbaum MS. Deformationbased morphometry and its relation to conventional volumetry of brain lateral ventricles in MRI. Neuroimage 2001;13:1140-5. https://doi.org/ 10.1006/nimg.2001.0771.

[48] Kurth F, Luders E, Gaser C. Voxel-based morphometry. Brain Mapping: An Encyclopedic Reference 2015;1:345-9. https://doi.org/10.1016/B978-0-12397025-1.00304-3.

[49] Fridgeirsson EA, Deng Z De, Denys D, van Waarde JA, van Wingen GA. Electric field strength induced by electroconvulsive therapy is associated with clinical outcome. Neurolmage Clin 2021;30:102581. https://doi.org/10.1016/ j.nicl.2021.102581.

[50] Dahnke R, Yotter RA, Gaser C. Cortical thickness and central surface estimation. Neuroimage 2013;65:336-48. https://doi.org/10.1016/ j.neuroimage.2012.09.050.

[51] Thielscher A, Opitz A, WIndhoff M. Impact of the gyral geometry on the electric dield induced by transcranaial magnetic stimulation. Neuroimage 2011;54:234-43.

[52] Basser PJ, Pajevic S, Pierpaoli C, Duda J, Aldroubi A. In vivo fiber tractography using DT-MRI data. Magn Reson Med 2000;44(4):625-32. https://doi.org/ 10.1002/1522-2594(200010)44:4<625::aid-mrm17>3.0.co;2-o.

[53] Leemans A, Jeurissen B, Sijbers J, Jones DK. ExploreDTI: a graphical toolbox for processing, analyzing, and visualizing diffusion MR data. In: Proceedings of the 17th scientific meeting. Honolulu, USA: International Society for Magnetic Resonance in Medicine; 2009. p. 3537.

[54] Güllmar D, Haueisen J, Reichenbach JR. Influence of anisotropic electrical conductivity in white matter tissue on the EEG/MEG forward and inverse 
solution. A high-resolution whole head simulation study. Neuroimage 2010;51:145-63. https://doi.org/10.1016/j.neuroimage.2010.02.014.

[55] Hammers A, Allom R, Koepp MJ, Free SL, Myers R, Lemieux L, et al. Threedimensional maximum probability atlas of the human brain, with particular reference to the temporal lobe. Hum Brain Mapp 2003;19:224-47. https:// doi.org/10.1002/hbm.10123.

[56] Lyden H, Espinoza RT, Pirnia T, Clark K, Joshi SH, Leaver a M, et al. Electroconvulsive therapy mediates neuroplasticity of white matter microstructure in major depression. Transl Psychiatry 2014;4:e380. https://doi.org/10.1038/ tp.2014.21.

[57] Nickl-Jockschat T, Palomero Gallagher N, Kumar V, Hoffstaedter F, Brügmann E, Habel U, et al. Are morphological changes necessary to mediate the therapeutic effects of electroconvulsive therapy? Eur Arch Psychiatr Clin Neurosci 2016;266:261-7. https://doi.org/10.1007/s00406-015-0631-z.

[58] Gryglewski G, Seiger R, Baldinger-Melich P, Unterholzner J, Spurny B, Vanicek T, et al. Changes in white matter microstructure after electroconvulsive therapy for treatment-resistant depression. Int $\mathrm{J}$ Neuropsychopharmacol 2020;23:20-5. https://doi.org/10.1093/ijnp/pyz059.

[59] Tosun D, Dabbs K, Caplan R, Siddarth P, Toga A, Seidenberg M, et al. Deformation-based morphometry of prospective neurodevelopmental changes in new onset paediatric epilepsy. Brain 2011;134:1003-14. https://doi.org/ 10.1093/brain/awr027.

[60] Blommaert J, Schroyen G, Vandenbulcke M, Radwan A, Smeets A, Peeters R, et al. Age-dependent brain volume and neuropsychological changes after chemotherapy in breast cancer patients. Hum Brain Mapp 2019;40(17): 4994-5010. https://doi.org/10.1002/hbm.24753.

[61] Sartorius A, Demirakca T, Böhringer A, Clemm von Hohenberg C, Aksay SS, Bumb JM, et al. Electroconvulsive therapy increases temporal gray matter volume and cortical thickness. Eur Neuropsychopharmacol 2016;26:506-17. https://doi.org/10.1016/j.euroneuro.2015.12.036.

[62] Sackeim HA. The impact of electroconvulsive therapy on brain grey matter volume: what does it mean? Brain Stimul 2020;13:1226-31. https://doi.org/ 10.1016/j.brs.2020.04.014.

[63] Wagstyl K, Lerch JP. Cortical thickness. In: Spaletta G, Piras F, Gili T, editors. Brain morphometry, vol. 136. New York, NY: Springer New York; 2018. p. 35-49.

[64] Bhardwaj RD, Curtis MA, Spalding KL, Buchholz BA, Fink D, Björk-Eriksson T, et al. Neocortical neurogenesis in humans is restricted to development. Proc Natl Acad Sci U S A 2006;103:12564-8. https://doi.org/10.1073/ pnas.0605177103.

[66] Lövdén M, Wenger E, Mårtensson J, Lindenberger U, Bäckman L. Structural brain plasticity in adult learning and development. Neurosci Biobehav Rev 2013;37:2296-310. https://doi.org/10.1016/j.neubiorev.2013.02.014.

[67] Wennström M, Hellsten J, Ekdahl CT, Tingström A. Electroconvulsive seizures induce proliferation of NG2-expressing glial cells in adult rat hippocampus. $\begin{array}{llll}\text { Biol Psychiatr 2003;54:1015-24. htps://doi.org/10.1016/ } & \text { 24 }\end{array}$ j.biopsych.2003.11.011.

[68] Jansson L, Wennström M, Johanson A, Tingström A. Glial cell activation in response to electroconvulsive seizures. Prog Neuro-Psychopharmacol Biol Psychiatry 2009;33:1119-28. https://doi.org/10.1016/j.pnpbp.2009.06.007.

[69] Sibson NR, Lowe JP, Blamire AM, Martin MJ, Obrenovitch TP, Anthony DC. Acute astrocyte activation in brain detected by MRI: new insights into T(1) hypointensity. J Cerebr Blood Flow Metabol 2008;28:621-32. https://doi.org/ 10.1038/sj.jcbfm.9600549.

[70] Franklin TR, Wang Z, Shin J, Jagannathan K, Suh JJ, Detre JA, et al. A VBM study demonstrating "apparent" effects of a single dose of medication on T1weighted MRIs. Brain Struct Funct 2013;218:97-104. https://doi.org/ 10.1007/s00429-012-0385-6.

[71] Briellmann RS, Wellard RM, Jackson GD. Seizure-associated abnormalities in epilepsy: evidence from MR imaging. Epilepsia 2005;46:760-6. https:// doi.org/10.1111/j.1528-1167.2005.47604.x.
[72] Hayasaka S, Nakamura M, Noda Y, Izuno T, Saeki T, Iwanari H, et al. Lateralized hippocampal volume increase following high-frequency left prefrontal repetitive transcranial magnetic stimulation in patients with major depression. Psychiatr Clin Neurosci 2017;71:747-58. https://doi.org/10.1111/pcn.12547.

[73] Baeken C, Wu GR, Sackeim HA. Accelerated iTBS treatment applied to the left DLPFC in depressed patients results in a rapid volume increase in the left hippocampal dentate gyrus, not driven by brain perfusion. Brain Stimul 2020;13:1211-7. https://doi.org/10.1016/j.brs.2020.05.015.

[74] Dalhuisen I, Ackermans E, Martens L, Mulders P, Bartholomeus J, de Bruijn A, et al. Longitudinal effects of rTMS on neuroplasticity in chronic treatmentresistant depression. Eur Arch Psychiatr Clin Neurosci 2021;271:39-47. https://doi.org/10.1007/s00406-020-01135-w.

[75] Perini GI, Toffanin T, Pigato G, Ferri G, Follador H, Zonta F, et al. Hippocampal gray volumes increase in treatment-resistant depression responding to vagus nerve stimulation. J ECT 2017;33:160-6. https://doi.org/10.1097/ YCT.0000000000000424.

[76] Enev M, McNally KA, Varghese G, Zubal IG, Ostroff RB, Blumenfeld H. Imaging onset and propagation of ECT-induced seizures. Epilepsia 2007;48:238-44. https://doi.org/10.1111/j.1528-1167.2007.00919.x.

[77] McNally KA, Blumenfeld $\mathrm{H}$. Focal network involvement in generalized seizures: new insights from electroconvulsive therapy. Epilepsy Behav 2004;5: 3-12. https://doi.org/10.1016/j.yebeh.2003.10.020.

[78] Bertram E. The relevance of kindling for human epilepsy. Epilepsia 2007;48(Suppl):65-74. https://doi.org/10.1111/j.1528-1167.2007.01068.x.

[79] Miyasaka M. Cortical and subcortical seizure discharge induced by bitemporal electroshock in cat. Folia Psychiatr Neurol Jpn 1959;13:113-23. https:/ doi.org/10.1111/j.1440-1819.1959.tb02426.x.

[80] Dabrowska N, Joshi S, Williamson J, Lewczuk E, Lu Y, Oberoi S, et al. Parallel pathways of seizure generalization. Brain 2019;142:2336-51. https://doi.org/ 10.1093/brain/awz170.

[81] Zheng L, Bin G, Zeng H, Zou D, Gao J, Zhang J, et al. Meta-analysis of voxelbased morphometry studies of gray matter abnormalities in patients with mesial temporal lobe epilepsy and unilateral hippocampal sclerosis. Brain Imaging Behav 2018;12:1497-503. https://doi.org/10.1007/s11682-0179797-5.

[82] Bower SPC, Vogrin SJ, Morris K, Cox I, Murphy M, Kilpatrick CJ, et al. Amygdala volumetry in "imaging-negative" temporal lobe epilepsy. J Neurol Neurosurg Psychiatry 2003;74:1245-9. https://doi.org/10.1136/jnnp.74.9.1245.

[83] Lv RJ, Sun ZR, Cui T, Guan HZ, Ren HT, Shao XQ. Temporal lobe epilepsy with amygdala enlargement: a subtype of temporal lobe epilepsy. BMC Neurol 2014;14:1-8. https://doi.org/10.1186/s12883-014-0194-z.

[84] Parent JM, Lowenstein DH. Seizure-induced neurogenesis: are more new neurons good for an adult brain? Prog Brain Res 2002;135:121-31. https:// doi.org/10.1016/S0079-6123(02)35012-X.

[85] Nakamura K, Ito M, Liu Y, Seki T, Suzuki T, Arai H. Effects of single and repeated electroconvulsive stimulation on hippocampal cell proliferation and spontaneous behaviors in the rat. Brain Res 2013;1491:88-97. https:// doi.org/10.1016/j.brainres.2012.10.052.

[86] Svensson M, Grahm M, Ekstrand J, Höglund P, Johansson M, Tingström A. Effect of electroconvulsive seizures on cognitive flexibility. Hippocampus 2016;26:899-910. https://doi.org/10.1002/hipo.22573.

[87] Vaidya VA, Siuciak JA, Du F, Duman RS. Hippocampal mossy fiber sprouting induced by chronic electroconvulsive seizures. Neuroscience 1999;89: 157-66. https://doi.org/10.1016/S0306-4522(98)00289-9.

[88] Ingram WM, Weston C, Lu WD, Hodge C, Poler SM, Nahi F, et al. Factors affecting electroconvulsive therapy ictal outcomes: duration and postictal suppression. AMIA Jt Summits Transl Sci Proc 2019:672-9. eCollection 2019.

[89] Luer B, Nobler MS, Moeller JR, Katzman GP, Prudic J, Devanand DP, et al. Quantitative EEG during seizures induced by electroconvulsive therapy: relation to treatment modality and clinical features. II. Topographic analyses. J ECT 2000;16:229-43. 